\title{
The Usefulness of $\mathrm{CO}_{2}$ Laser-assisted Phonomicrosurgery Using a Computer-guided Scanner in Benign Vocal Fold Mucosal Disorders
}

Young Ho Ju

Kwang-Yoon Jung

Soon-Young Kwon

Jeong-Soo Woo

Jae-Gu Cho

Min Woo Park

Seung-Kuk Baek

Department of Otolaryngology-Head and Neck Surgery, Korea University College of Medicine, Seoul, Korea
Received November 7, 2012

Reviced November 22, 2012

Accepted November 23, 2012

\section{Correspondence}

Seung-Kuk Baek

Department of Otolaryngology-Head and Neck Surgery, Korea University College of Medicine, Anam-dong 5-ga 126-1, Seongbuk-gu,

Seoul 136-705, Korea

Tel: +82-2-920-5486

Fax: +82-2-925-5233

E-mail: mdskbaek@gmail.com

(C) Korean Society for Laser Medicine and Surgery

(c) This is an open access article distributed under the terms of the Creative Commons Attribution NonCommercial License (http://creativecommons.org/ licenses/by-nc/3.0) which permits unrestricted noncommercial use, distribution, and reproduction in any medium, provided the original work is properly cited.

\section{Background and Objectives}

Our aim in this study is to evaluate the efficacy and usefulness of $\mathrm{CO}_{2}$ laser-assisted phonomicrosurgery using a computer-guided scanner (CGS) in benign vocal fold mucosal disorders.

\section{Materials and Methods}

The current study enrolled 93 patients who underwent phonomicrosurgery for benign vocal fold mucosal disorders. Twenty five patients underwent $\mathrm{CO}_{2}$ laser-assisted phonomicrosurgery using CGS (Group 1), and 68 patients underwent conventional phonomicrosurgery using a cold instrument (Group 2).

\section{Results}

All voice analysis parameters showed significant improvement after surgical treatment, except fundamental frequency. Differences between preoperative and postoperative parameters of each voice analysis data did not differ significantly between the two groups.

\section{Conclusion}

Although no significant difference was observed between the two groups, $\mathrm{CO}_{2}$ laser assisted phonomicrosurgery using CGS allows for performance of more accurate and delicate incision with a controllable depth of beam penetration.

\section{Key words}

Phonomicrosurgery; Computer-guided scanner; $\mathrm{CO}_{2}$ laser 


\section{INTRODUCTION}

Diverse treatments have been recommended for benign vocal fold mucosal disorders, including medical therapy, voice therapy and phonomicrosurgery alone or combined. Phonomicrosurgery has been performed with various instruments such as the carbon dioxide $\left(\mathrm{CO}_{2}\right)$ laser. Since the $\mathrm{CO}_{2}$ laser was introduced to medical community, it quickly gained popularity among otolaryngologists.

In the late 1980s and early 1990s, a majority of studies has focused the safety of the $\mathrm{CO}_{2}$ laser in phonomicrosurgery. ${ }^{1}$ The early $\mathrm{CO}_{2}$ laser had some problems in relationship to the effects on the lesion and the surrounding tissues and the potential for vocal fold scarring. ${ }^{2}$ The development of micromanipulator for the $\mathrm{CO}_{2}$ laser has made it possible to create much smaller beam diameter and little collateral thermal tissue interaction when compared with the other medical lasers, including neodymium:yttrium-aluminumgarnet (Nd:YAG) laser, argon laser, or potassium titanyl phosphate (KTP) laser. ${ }^{3,4}$

A new Computer-guided scanner (CGS) recently applied to the $\mathrm{CO}_{2}$ laser micromanipulator allows the beam to sweep a given surface with extreme rapidity. Such a rapid beam sweep makes controllable-penetrating depth of the $\mathrm{CO}_{2}$ laser possible. ${ }^{5}$

However, no systematized study of the efficacy of $\mathrm{CO}_{2}$ laser using CGS has been reported yet. The purpose of this study is to evaluate the efficacy and usefulness of $\mathrm{CO}_{2}$ laser-assisted phonomicrosurgery using CGS in benign vocal fold mucosal disorders.

\section{MATERIALS AND METHODS}

\section{Study design}

The study group consisted of 107 patients with benign vocal fold mucosal disorder who underwent phonomicrosurgery at Otolaryngology-Head and Neck Surgery, Anam Hospital, Korea University, Seoul, Korea, between September 2010 to May 2012. We retrospectively selected patients on the basis of medical records. Subjects were excluded due to the followings: insufficient postoperative evaluation and management, irregular follow up period, and young age before 20 years. Of the 107 patients eligible for the present study, 14 patients were excluded prior to enrollment. Thus, a total of 93 patients were included in the present study, 25 patients underwent the $\mathrm{CO}_{2}$ laser-assisted phonomicrosurgery using CGS (Group 1), and 68 patients underwent the conventional phonomicrosurgery using cold instrument
(Group 2). All subjects were given informed consent. The institutional review board of our institution approved the protocol of our study.

\section{Surgery and vocal rehabilitation}

The timing of surgery was individualized according to the patient's preference, lesion characteristics, and concomitant problems, such as poor vocal hygiene. All of the patients received behavioral intervention for vocal hygiene before the surgical treatment.

All of the surgical procedures were performed under general anesthesia by the same surgeon. The operation was aimed at maximally preserving the vocal fold's layered microstructure, including the lamina propria and epithelium. In Group 1, $\mathrm{CO}_{2}$ laser with CGS (Acublade ${ }^{\mathrm{TM}}$ system; Lumenis, Santa Clara, CA, USA) was used for the incision and dissection processes. The laser settings used were 2-mm beam length, 200- $\mu \mathrm{m}$ penetration, and the superpulsed wave in repeated pulse mode. Curved incision was performed to avoid injuring the vocal ligament while maintaining the free edge of the vocal fold taut with micro-forceps. The conventional phonomicrosurgery using the micro-scissors and microknife was performed in Group 2.

After surgery, absolute voice rest was recommended for the first 7 days. For 2 to 3 weeks after surgery, the patients were instructed to increase their daily phonation time gradually.

\section{Voice analysis}

The subjects were recorded two times: before surgery and 2 months after the operation. The following variables were measured: acoustic analysis (fundamental frequency, jitter, shimmer, noise-to-harmonic ratio), aerodynamic analysis (mean flow rate, maximal phonation time, subglottic pressure), GRBAS scale Igrade, breathiness, roughness, strain, asthenicity, aphonial and voice handicap index (VHI). Acoustic variables were measured using the Multi-Dimensional Voice Program (MDVP) from the Computerized Speech Lab (CSL), Model 4500 (KayPENTAX, Lincoln Park, NJ, USAl for a few seconds of the sustained phonation of /a/. The subjective estimation of voice quality was performed using the GRBAS scale designed by Hirano and De Bodt et al. ${ }^{6}$ The scale was administered by an experienced phonetician who had no information about the study design. The VHI consists of 30 questions divided by content into 3 categories: functional, physical, and emotional parameters. All of the patients completed the $\mathrm{VHI}$ questionnaires using a 5-point rating scale to indicate 
their response. The scale is ordinal and is scored from 0 (Never) to 4 (Always) for each of the questions, with a minimum score of 0 and a maximum score of 120 . Higher scores indicate a more severe perception of disability because of the voice problem.

\section{Voice therapy}

All of the patients in the study group underwent two voice therapy sessions lapproximately 30 minutes per session) in the fourth week after laryngeal microsurgery. Voice therapy included the general approach toward managing vocal hygiene, including voice rest, adequate hydration, the reduction/elimination of laryngeal irritants, the reduction of vocal abuse and hard glottal attacks, the reduction of vocal loudness and speech rate, and the elimination of chronic throat clearing and coughing. Resonance voice exercises, such as humming and relaxation exercises that aimed to release musculoskeletal tension in the shoulder and neck, were

Table 1. Demographics of enrolled patients

\begin{tabular}{lccc}
\hline & Group 1 (N=25) & Group 2 (N=68) & $p$-value \\
\hline Sex (\%) & & & 0.185 \\
Female & $9(36)$ & $35(51.5)$ & \\
$\quad$ Male & $16(64)$ & $33(48.5)$ & \\
Age & 49.6 & 49.2 & 0.406 \\
Occupation related voice overuse & & & 0.129 \\
$\quad$ Yes & $9(36)$ & $11(16.2)$ & \\
No & $16(64)$ & $57(83.8)$ & \\
\hline
\end{tabular}

Group 1, $\mathrm{CO}_{2}$ laser-assisted phonomicrosurgery using computerguided scanner; Group 2, phonomicrosurgery using cold instruments.

Table 2. Effect of treatment on voice analysis in the Group $1\left(\mathrm{CO}_{2}\right.$ laser-assisted phonomicrosurgery using computer-guided scanner)

\begin{tabular}{lccr}
\hline & \multicolumn{2}{c}{ Group 1 (N=25) } & \\
\cline { 2 - 3 } & Preoperative & Postoperative & \\
\hline Aerodynamic analysis & & & \\
MFR & $254.00 \pm 150.64$ & $157.20 \pm 71.33$ & $<0.01$ \\
MPT & $12.70 \pm 5.42$ & $17.22 \pm 5.78$ & $<0.01$ \\
Psub & $14.32 \pm 6.17$ & $10.53 \pm 4.13$ & $<0.01$ \\
Efficiency & $140.42 \pm 134.74$ & $288.81 \pm 258.31$ & $<0.01$ \\
Acoustic analysis & & & \\
Fundamental frequency & $148.54 \pm 35.43$ & $152.99 \pm 44.24$ & 0.54 \\
Jitter & $2.64 \pm 2.43$ & $1.18 \pm 0.88$ & $<0.01$ \\
Shimmer & $6.46 \pm 4.45$ & $3.05 \pm 1.55$ & $<0.01$ \\
NHR & $0.18 \pm 0.08$ & $0.13 \pm 0.02$ & $<0.01$ \\
\hline
\end{tabular}

MFR, mean flow rate; MPT, maximal phonation time; Psub, subglottic pressure; NHR, noise harmony ratio. also taught.

\section{Statistics}

Paired or independent $t$ tests were used to compare the voice outcomes and questionnaire scores before and after surgery and between Group 1 and 2. For all tests, a probability of $<0.05$ was accepted as statistically significant. All of the statistical analyses were performed using SPSS software version 12.0 (SPSS Inc., Chicago, IL, USA).

\section{RESULTS}

\section{Demographics}

The mean age was 49.6 years in Group 1 and 47.2 years in Group 2. Patients' various occupational fields were

Table 3. Effect of treatment on voice analysis in the Group 2 (phonomicrosurgery using cold instruments)

\begin{tabular}{lccr}
\hline & \multicolumn{2}{c}{ Group 2 (N=68) } & \multirow{2}{c}{-value } \\
\cline { 2 - 3 } & Preoperative & Postoperative & \\
\hline Aerodynamic analysis & & & \\
MFR & $234.71 \pm 124.28$ & $159.12 \pm 79.96$ & $<0.01$ \\
MPT & $10.52 \pm 5.17$ & $14.57 \pm 5.46$ & $<0.01$ \\
Psub & $13.25 \pm 5.21$ & $10.71 \pm 3.04$ & $<0.01$ \\
Efficiency & $135.86 \pm 148.32$ & $213.36 \pm 215.78$ & $<0.01$ \\
Acoustic analysis & & & \\
Fundamental frequency & $166.54 \pm 47.82$ & $172.52 \pm 46.05$ & 0.11 \\
Jitter & $2.67 \pm 1.87$ & $1.08 \pm 1.73$ & $<0.01$ \\
Shimmer & $7.62 \pm 5.54$ & $3.70 \pm 5.68$ & $<0.01$ \\
NHR & $0.17 \pm 0.10$ & $0.13 \pm 0.05$ & $<0.01$ \\
\hline
\end{tabular}

MFR, mean flow rate; MPT, maximal phonation time; Psub, subglottic pressure; NHR, noise harmony ratio.

Table 4. Comparing the voice outcomes between the Group 1 and 2

\begin{tabular}{lccc}
\hline \multicolumn{1}{c}{ Difference } & Group 1 (N=25) & Group 2 (N=68) & $p$-value \\
\hline Aerodynamic analysis & & & \\
$\quad \Delta$ MFR & -96.8 & -75.59 & 0.40 \\
$\Delta$ MPT & 4.52 & 4.05 & 0.70 \\
$\Delta$ Psub & -3.79 & -2.55 & 0.34 \\
$\quad \Delta$ Efficiency & 148.39 & 77.49 & 0.15 \\
Acoustic analysis & & & \\
$\quad \Delta$ Fundamental frequency & 4.44 & 5.98 & 0.84 \\
$\Delta$ Jitter & -1.47 & -1.59 & 0.82 \\
$\Delta$ Shimmer & -3.42 & -3.92 & 0.74 \\
$\Delta$ NHHR & -0.05 & -0.05 & 0.81 \\
\hline
\end{tabular}

Difference, postoperative parameters-preoperative parameters; MFR, mean flow rate; MPT, maximal phonation time; Psub, subglottic pressure; NHR, noise harmony ratio. 
divided into two groups: occupation-related voice overuse group, such as teachers, counselors, and salespersons, and no overuse group. All of baseline demographics were not significantly different between the two groups (Table 1).

\section{Effectiveness of phonomicrosurgery}

In both groups, most of the voice analysis parameters were significantly improved after surgical treatment and postoperative voice therapy except fundamental frequency (Table 2 and 3).

\section{$\mathrm{CO}_{2}$ laser-assisted phonomicrosurgery using CGS or cold instrument}

The differences between preoperative and postoperative parameter of each voice analysis data (postoperative parameter minus preoperative parameter) were calculated and compared between two groups to estimate the usefulness of $\mathrm{CO}_{2}$ laser-assisted phonomicrosurgery with CGS. There was no significant difference in voice analysis data (Table 4).

\section{DISCUSSION}

The $\mathrm{CO}_{2}$ laser as one of the gas lasers emits electromagnetic energy as a wavelength of $10.600 \mu \mathrm{m}$, which has a high coefficient of absorption for water. The advantage of $\mathrm{CO}_{2}$ laser is that it induces little collateral thermal damage to surrounding tissue. Furthermore, the thermal penetration of the $\mathrm{CO}_{2}$ laser ranges in microns, whereas that of the other lasers ranges in millimeters. ${ }^{3,7}$

The $\mathrm{CO}_{2}$ laser possesses some physiological and technical advantages. It influences not only vaporization of fluid in the tissue instantly but also ignition and scattering of the solid element by the pressure difference resulting from vaporization. ${ }^{8}$ In addition, this laser offers surgeons an opportunity for maximal operative field and easier instrumentation. Decreased risk of postoperative bleeding, increased sterility and better hemostatic effect are also potential benefits. ${ }^{9}$ All of these factors make the $\mathrm{CO}_{2}$ laser-assisted phonomicrosurgery a useful therapeutic tool for the treatment of benign vocal fold mucosal disorders.

The micromanipulator, connecting the operating microscope and the laser arm, yields the beam diameter with a very small size of $250 \mu \mathrm{m}$. Therefore, this micromanipulator makes possible the more accurate incision and dissection of tissue required for phonomicrosurgery. ${ }^{10-12}$ However, single pulse dissection using the Acuspot micromanipulator does not attain an incision as regular as that achieved with micro-scissors.
The $\mathrm{CO}_{2}$ laser with CGS makes it possible for the scanner-assisted beam to travel across the target as a straight or curved incision line as regular as the incision obtained with the micro-scissors. In addition, the length and penetration depth of laser beam can be adjusted.

In the present study, we evaluated the usefulness of the $\mathrm{CO}_{2}$ laser with CGS by comparing the patients who underwent the $\mathrm{CO}_{2}$ laser assisted phonomicrosurgery using CGS with the patients who underwent the conventional phonomicrosurgery using cold instrument. Although there was no significant difference between the two groups, the $\mathrm{CO}_{2}$ laser assisted phonomicrosurgery using CGS provided an incision as regular as cold instrumentations such as micro-scissors, while achieving hemostasis unattainable with cold instrumentations.

In conclusion, the CGS is the most effective and useful tool for the $\mathrm{CO}_{2}$ laser-assisted phonomicrosurgery. It helps achieve more accurate and delicate incision with the controllable depth of beam penetration. Additionally, since the beam shape is adjustable, lineal and curved incision and round ablation are possible.

\section{REFERENCES}

1. Sataloff RT, Spiegel JR, Hawkshaw M, Jones A. Laser surgery of the larynx: the case for caution. Ear Nose Throat J 1992;71: 593-5.

2. Benninger MS. Laser surgery for nodules and other benign laryngeal lesions. Curr Opin Otolaryngol Head Neck Surg 2009; 17:440-4.

3. Lesinski SG, Palmer A. Lasers for otosclerosis: $\mathrm{CO}_{2}$ vs. Argon and KTP-532. Laryngoscope 1989;99(6 Pt 2 Suppl 46):1-8.

4. Polanyi TG, Bredemeier HC, Davis TW Jr. A CO 2 laser for surgical research. Med Biol Eng 1970;8:541-8.

5. Remacle M, Hassan F, Cohen D, Lawson G, Delos M. New computer-guided scanner for improving $\mathrm{CO}_{2}$ laser-assisted microincision. Eur Arch Otorhinolaryngol 2005;262:113-9.

6. De Bodt MS, Wuyts FL, Van de Heyning PH, Croux C. Testretest study of the GRBAS scale: influence of experience and professional background on perceptual rating of voice quality. J Voice 1997;11:74-80.

7. Reinisch L. Laser physics and tissue interactions. Otolaryngol Clin North Am 1996;29:893-914.

8. Yan Y, Olszewski AE, Hoffman MR, Zhuang P, Ford CN, Dailey $\mathrm{SH}$, et al. Use of lasers in laryngeal surgery. J Voice 2010;24: 102-9.

9. Reinisch L, Ossoff RH. Laser applications in otolaryngology. Otolaryngol Clin North Am 1996;29:891-2.

10. Keilmann A, Biermann G, Hörmann K. $\mathrm{CO}_{2}$ laser versus conventional microlaryngoscopy in benign changes of the vocal 


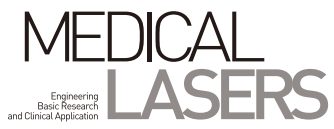

cords. Laryngorhinootologie 1997;76:484-9.

11. Remacle M, Lawson G, Watelet JB. Carbon dioxide laser microsurgery of benign vocal fold lesions: indications, techniques, and results in 251 patients. Ann Otol Rhinol
Laryngol 1999;108:156-64.

12. Remacle M, Lawson G, Degols JC, Evrard I, Jamart J. Microsurgery of sulcus vergeture with carbon dioxide laser and injectable collagen. Ann Otol Rhinol Laryngol 2000;109:141-8. 